\title{
Analytical Report of Psychological Status of 130 Frontline Medical Staff Dealing With COVID-19
}

\author{
Wang Lijuan $^{1 \#}$, Lu Longxi ${ }^{2 \#}$, Zheng Jisheng ${ }^{1}$, Liu Xiaozheng ${ }^{3}$, Zhu Ruichen ${ }^{4}$ and Guo Zhongwei ${ }^{1^{*}}$ \\ ${ }^{1}$ Department of Psychiatry, Tongde Hospital of Zhejiang Province and Mental Health Center of \\ Zhejiang Province, China \\ ${ }^{2}$ Zhejiang Province Center for Disease Control and Prevention, China \\ ${ }^{3}$ Department of Radiology of the Second Affiliated Hospital, Yuying Children's Hospital, China- \\ USA Neuroimaging Research Institute, Wenzhou Medical University, China \\ ${ }^{4}$ Zhejiang Hospital, China

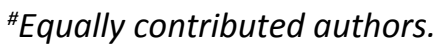

*Corresponding author: Guo Zhongwei, Department of Psychiatry, Tongde Hospital of Zhejiang Province and Mental Health Center of Zhejiang Province, Zhejiang, 310012, China, Tel: +86-0571-89972458; +8613588448303

\begin{abstract}
Background: China, especial Wuhan city, has been severely affected by COVID-19. This study aimed to understand the psychological status and related factors of frontline medical staff in the COVID-19 epidemic.

Methods: Participants were 130 first-line medical staff working in Tianyou Hospital, Wuhan. Participants completed the Generalized Anxiety Disorder-7 scale and the Patient Health Questionnaire-9.

Findings: 1) A total of 48 participants (36.9\%) showed emotional symptoms, $41(31.8 \%)$ showed anxiety symptoms, and 36 (27.7\%) showed depressive symptoms. 2) Females showed more severe anxiety than males $(P<0.05)$; intermediate level staff showed greater anxiety than junior and senior staff $(P<0.05)$. 3) Disease factors, work factors, time factors, and safety factors were all related to anxiety and depression $(P<0.05)$.

Interpretation: There is a high incidence of emotional symptoms in frontline medical staff in the COVID-19 epidemic. Emotional response is closely related to the characteristics of the epidemic. Psychological interventions are necessary to manage these symptoms.
\end{abstract}

\section{Keywords}

COVID-19, Medical staff, Psychology, Anxiety, Depression

\section{Introduction}

The COVID-19 epidemic is considered a public health emergency of international concern owing to its rapid spread, extremely harmful effects, and pathogenic complexity [1]. Most confirmed cases and deaths worldwide have occurred in Wuhan, the source area of the COVID-19 epidemic. All movements in Wuhan were temporarily restricted from January 23,2020 . There were 68,500 confirmed cases up to February 15, 2020, and the number continued to substantially increase, inevitably causing the public psychological stress. On January 26, the Chinese government issued guidelines for emergency psychological crisis interventions, as an important part of epidemic prevention and control.

Medical staffs are always at the forefront of the fight against epidemics, and are in close contact with patients and toxins. Increased risk of infection, heavy workload, witnessing patient pain and distress, and isolation between colleagues greatly increase psychological stress in medical staff. It is important to acknowledge that the mental health problems of medical staff not only affect the quality of their work in epidemic control, but also lead to psychological trauma even after the epidemic has passed [2]. Therefore, maintaining the

Citation: Lijuan W, Longxi L, Jisheng Z, Xiaozheng L, Ruichen Z, et al. (2020) Analytical Report of Psychological Status of 130 Frontline Medical Staff Dealing With COVID-19. Int J Depress Anxiety 3:021. doi. org/10.23937/2643-4059/1710021

Received: May 03, 2020: Accepted: June 08, 2020: Published: June 10, 2020

Copyright: (C) 2020 Lijuan W, et al. This is an open-access article distributed under the terms of the Creative Commons Attribution License, which permits unrestricted use, distribution, and reproduction in any medium, provided the original author and source are credited. 
mental health of medical staff is an important part of the response to the COVID-19 epidemic.

Previous studies have shown that the psychological stress responses of medical staff vary across different epidemics, and even in staff dealing with the same epidemic in different regions. For example, Wang, et al. reported that during the SARS epidemic, the main emotional reactions of medical staff in Wuhan were anxiety and depression [3]; these reactions were related to differences in gender and social support. Medical staff at Xiaotangshan hospital, China, dealing with the SARS epidemic showed obsessive-compulsive, interpersonal sensitivity, depression, and somatization psychological stress responses; influencing factors of these responses were coping style, social flexibility, evaluation of work, and the overall impact of living with SARS [4]. During the human H7N9 avian influenza epidemic, the emotional symptoms of medical staff in Zhejiang Province were fear, neurasthenia, depression, anxiety, and hypochondriasis; these symptoms positively correlated with subjects' risk perception level [5].

In the COVID-19 epidemic, tens of thousands of medical staff have provided emergency assistance in Wuhan. They must adapt to a different regional environment, a new mode of working team cooperation. Moreover, they are at constant high risk of infection. Therefore, medical staffs face more stressors than the general public. In this study, we focused on the psychological state of medical staff from Zhejiang Province during the COVID-19 epidemic by investigating anxiety and depression severity. We also explored the effect of relevant influencing factors on psychological state. We hope to provide a scientific basis for targeted interventions for psychological distress in such staff.

\section{Method}

\section{Investigatory method}

Using the Questionnaire Star technology platform, we designed a questionnaire and distributed it to 150 medical staff using the WeChat app. Participants submitted completed questionnaires online anonymously. Participation was completely voluntary and participants received no payment for taking part in the study.

\section{Questionnaire measures}

The questionnaire comprised 13 questions and could be completed in 2 minutes. The variables assessed were as follows: 1) General characteristics: Gender, age, marital status, educational background, occupation, professional title, department of origin, time spent dealing with the epidemic; 2) General psychological state; 3) The Generalized Anxiety Scale-7 (GAD-7) was used to assess anxiety symptoms [6]. The GAD-7 is a 7-item self-assessed scale rated on a 4-point Likert scale; the total possible score is 21 . Scores $<5$ indicate no anxiety symptoms and scores $\geq 5$ indicate the presence of anxiety symptoms: Scores 5-9 indicate mild anxiety tendency, 10-13 indicate moderate anxiety tendency, and $\geq 14$ indicate severe anxiety tendency. 4) The Patient Health Questionnaire-9 (PHQ-9) depression self-assessment scale was used to assess depression symptoms [7]. PHQ-9 is a 9-item self-assessment scale rated on a 4-point Likert scale; higher scores indicate more severe depression. A total score $<5$ indicates no depression and $\geq 5$ indicates depression: Scores 5-9 indicate mild depression, 10-14 moderate depression, 15-19 moderate and severe depression, and 20-27 severe depression. 5) A simple self-designed questionnaire was used to assess related factors; this comprised eight items: Disease factors (e.g., fear of disease), work factors (e.g., high work intensity), time factors (e.g., no clear end point), family factors (e.g., complaints from family), safety assurance (e.g., lack of protective equipment), management factors (e.g., inadequate efforts of current management), human support factors (e.g., insufficient care), and other factors. Each item had two response options: "No" (0 points) and "Yes" (1 point).

\section{Statistical methods}

The data were exported from the Questionnaire Star platform and saved in Excel format. SPSS 19.0 software was used to establish a database for analysis. The $\chi^{2}$ test was used to examine differences in the incidence of emotional symptoms by different demographic characteristics. Pearson correlation analysis was used to examine associations between emotional symptoms and influencing factors. $P<0.05$ was considered to indicate statistical significance.

\section{Results}

\section{General demographic characteristics of partici- pants with emotional symptoms}

A total of 130 questionnaires were collected, a response rate of $86.7 \%$. A total of 48 participants (36.9\%) had emotional symptoms (anxiety/depression), 41 (31.8\%) had anxiety symptoms, and 36 had depression symptoms $(27.7 \%)$ (Table 1$)$.

\section{The severity of emotional symptoms by different demographic characteristics}

There were 30 patients with mild anxiety, 4 with moderate anxiety, and 7 with severe anxiety. The median (P50) $25^{\text {th }}$ percentile-75 $5^{\text {th }}$ percentile (P25-P75) GAD-7 score was 7.00 (5.00-11.00). There were 23 patients with mild depression, 7 with moderate depression, 6 with severe depression. The P50 (P25-P75) of PHQ-9 score was 7.50 (5.25-11.75). Females showed more severe anxiety than males $(H=0.199, P<0.05)$; intermediate level staff showed greater anxiety than junior or senior staff $(\mathrm{H}=5.775, P<0.05)$ (Table 2$)$. 
Table 1: General demographic characteristics of participants with emotional symptoms.

\begin{tabular}{|c|c|c|c|}
\hline Item & $\begin{array}{l}\text { Total } \\
(n=130)\end{array}$ & $\begin{array}{l}\text { Anxiety } \\
(n=41)\end{array}$ & $\begin{array}{l}\text { Depression } \\
(n=36)\end{array}$ \\
\hline \multicolumn{4}{|l|}{ Gender } \\
\hline Male & 45 & $16(35.6 \%)$ & $10(22.2 \%)$ \\
\hline Female & 85 & $25(29.4 \%)$ & $26(30.6 \%)$ \\
\hline \multicolumn{4}{|l|}{ Age } \\
\hline $20-30$ & 27 & $16(59.3 \%)$ & $9(33.3 \%)$ \\
\hline $31-40$ & 81 & $23(28.4 \%)$ & $23(28.4 \%)$ \\
\hline$>40$ & 22 & $6(27.3 \%)$ & $4(18.2 \%)$ \\
\hline \multicolumn{4}{|l|}{ Marriage } \\
\hline Unmarried & 30 & $10(33.3 \%)$ & $9(30 \%)$ \\
\hline Married & 100 & $31(31 \%)$ & $27(27 \%)$ \\
\hline \multicolumn{4}{|l|}{ Education } \\
\hline Specialty & 5 & $3(60 \%)$ & $4(80 \%)$ \\
\hline Undergraduate & 109 & $33(30.3 \%)$ & $29(26.6 \%)$ \\
\hline $\begin{array}{l}\text { Master degree } \\
\text { or above }\end{array}$ & 16 & $5(31.3 \%)$ & $3(18.8 \%)$ \\
\hline \multicolumn{4}{|l|}{ Occupation } \\
\hline Doctor & 39 & $10(25.7 \%)$ & $9(23.1 \%)$ \\
\hline Nurse & 86 & $28(32.6)$ & $26(30.2 \%)$ \\
\hline Management & 5 & $3(60 \%)$ & $1(20 \%)$ \\
\hline \multicolumn{4}{|l|}{ Title } \\
\hline Primary & 36 & $16(44.4 \%)$ & $14(8.9 \%)$ \\
\hline Intermediate & 66 & $16(24.2 \%)$ & $15(22.7 \%)$ \\
\hline Senior & 28 & $9(32.1 \%)$ & $7(25 \%)$ \\
\hline \multicolumn{4}{|c|}{ Original department } \\
\hline $\begin{array}{l}\text { Infectious } \\
\text { disease } \\
\text { department }\end{array}$ & 15 & $5(33.3 \%)$ & $7(46.7 \%)$ \\
\hline $\begin{array}{l}\text { Department of } \\
\text { respiration }\end{array}$ & 41 & $12(29.3 \%)$ & $10(24.4 \%)$ \\
\hline ICU & 49 & $14(28.6 \%)$ & $11(22.4 \%)$ \\
\hline Other & 25 & $10(40 \%)$ & $8(32 \%)$ \\
\hline \multicolumn{4}{|c|}{ Time of anti epidemic } \\
\hline$<1$ week & 12 & $5(41.7 \%)$ & $3(25 \%)$ \\
\hline $1 \sim 2$ weeks & 113 & $34(30.1 \%)$ & 32 (28.3\%) \\
\hline$>2$ weeks & 5 & $2(40 \%)$ & $1(20 \%)$ \\
\hline
\end{tabular}

\section{Correlation between emotional symptoms and self-assessed factors}

The results showed that anxiety was positively correlated with disease factors $(r=0.3364, P<0.001)$, work factors $(r=0.4134, P<0.001)$, time factors $(r=0.2773$, $P=0.0015)$, safety assurance $(r=0.2589, P=0.0031)$, and management factors $(r=0.2758, P=0.0016)$. Anxiety was negatively correlated with other factors ( $r$ $=-0.3145, P<0.001)$. Depression was positively correlated with disease factors $(r=0.3848, P<0.001)$, work factors $(r=0.3625, P<0.001)$, time factors $(r=0.2479$, $P=0.0046)$, safety assurance $(r=0.2227, P=0.0112)$, and management factors $(r=0.2216, P=0.0116)$. De- pression was negatively correlated with other factors ( $r$ $=-0.2872, P=0.001)$.

\section{Discussion}

The present findings show that about one-third of the first line medical staff caring for patients with COVID-19 has an emotional response. This is lower than the incidence of emotional responses of Chinese medical staff in Africa during the Ebola virus epidemic $[8,9]$. $\mathrm{Ji}$, et al. evaluated the psychological symptoms of 161 participants in an Ebola treatment center in Jui Government Hospital, Sierra Leone, in 2015 [9]. The incidence of psychological symptoms of Chinese medical staff was $72.2 \%$. Liu, et al. also reported that $81.82 \%$ of frontline medical staff working at Sierra Leone Friendship Hospital in 2014 showed anxiety symptoms [10]. Chinese medical staff working in such conditions may experience psychological distress not only because of high-risk exposure, the breakdown of social support systems, and working in a different culture, but also because of shortage of supplies $[11,12]$. The incidence of emotional responses in our current survey was also lower than that reported during the SARS epidemic in 2003 [13], the H1N1 pandemic in 2009 [14,15], and the H7N9 epidemic in 2013 [5]. The main reason for the difference may be the effect of the valuable experience gained from managing SARS, H1N1, and other major infectious diseases. A recent mental health survey of medical staff caring for patients with COVID-19 at Nanfang Hospital, Southern Medical University, showed that $50.7 \%$ had depression and $44.7 \%$ had anxiety [16], incidences higher than the present ones. A possible explanation for the difference may be regional factors [17]. However, more attention should be paid to the mental health of medical staff because the "challenge of managing COVID-19" is far from over [18].

Gender affects the severity of anxiety symptoms. We found that females experienced more severe anxiety than males, as in previous studies $[19,20]$. Lee and Tang reported that female participants who had worked through SARS or H7N9 epidemics showed higher Post-Traumatic Stress Disorder (PTSD) levels than males $[19,20]$. Several studies $[21,22]$ have shown that gender is closely related to anxiety, and females were more likely to use avoidance behaviours during the H1N1 pandemic [22]. Women are at higher risk of PTSD than men after exposure to similar traumatic events, and the association between gender and PTSD is independent of mechanism and injury event-related factors such as perceived threat to life [23]. This sex difference may be a result of sex differences in perceptions of stressors $[14,22,24]$.

Our results indicate that, of the three professional groups, intermediate staff showed the most severe anxiety symptoms, suggesting that professional level may be another factor that affects anxiety severity. Tang, et al. investigated the influencing factors of PTSD 
Table 2: Severity of emotional symptoms by different demographic characteristics (P50 [P25-P75]).

\begin{tabular}{|c|c|c|}
\hline Item & GAD-7 & PHQ-9 \\
\hline \multicolumn{3}{|l|}{ Gender } \\
\hline Male & $5.00(5.00 \sim 7.00)$ & $7.50(5.75 \sim 10.50)$ \\
\hline Female & $7.00(6.00 \sim 12.00)$ & $7.50(5.00 \sim 12.50)$ \\
\hline $\mathrm{H}$ value & -0.199 & -0.321 \\
\hline$P$ value & $0.046^{*}$ & 0.748 \\
\hline \multicolumn{3}{|l|}{ Age } \\
\hline $20-30$ & $5.50(5.00 \sim 7.75) /$ & $6.00(5.00 \sim 9.50)$ \\
\hline $31-40$ & $7.00(5.00 \sim 15.00)$ & $8.00(6.00 \sim 14.00)$ \\
\hline$>40$ & $7.50(5.00 \sim 12.50)$ & $7.00(5.00 \sim 12.00)$ \\
\hline $\mathrm{H}$ value & 2.62 & 4.354 \\
\hline$P$ value & 0.27 & 0.113 \\
\hline \multicolumn{3}{|l|}{ Marriage } \\
\hline Unmarried & $6.00(5.00 \sim 8.25)$ & $6.00(5.00 \sim 9.50)$ \\
\hline Married & $7.00(5.00 \sim 13.00)$ & $8.00(6.00 \sim 13.00)$ \\
\hline $\mathrm{H}$ value & -1.166 & -1.552 \\
\hline$P$ value & 0.243 & 0.127 \\
\hline \multicolumn{3}{|l|}{ Education } \\
\hline Specialty & $5.00(5.00 \sim 7.00)$ & $7.00(5.00 \sim 9.75)$ \\
\hline Undergraduate & $7.00(5.00 \sim 11.00)$ & $7.00(5.50 \sim 12.00)$ \\
\hline Master degree or above & $6.00(5.00 \sim 9.50)$ & $9.00(8.50 \sim 11.00)$ \\
\hline $\mathrm{H}$ value & 1.65 & 1.533 \\
\hline$P$ value & 0.438 & 0.465 \\
\hline \multicolumn{3}{|l|}{ Occupation } \\
\hline Doctor & $6.00(5.00 \sim 7.50)$ & $6.00(5.00 \sim 8.50)$ \\
\hline Nurse & $7.00(5.00 \sim 11.00)$ & $9.00(6.00 \sim 12.00)$ \\
\hline Management & $6.00(5.50 \sim 12.50)$ & I \\
\hline $\mathrm{H}$ value & 1.22 & -0.318 \\
\hline$P$ value & 0.543 & 0.188 \\
\hline \multicolumn{3}{|l|}{ Title } \\
\hline Primary & $5.50(5.00 \sim 7.75)$ & $6.00(5.00 \sim 10.25)$ \\
\hline Intermediate & $10.00(6.00 \sim 17.00)$ & $6.00(5.00 \sim 11.00)$ \\
\hline Senior & $7.00(5.00 \sim 8.00)$ & $7.00(5.00 \sim 9.00)$ \\
\hline $\mathrm{H}$ value & 5.775 & 5.396 \\
\hline$P$ value & $0.049^{*}$ & 0.067 \\
\hline \multicolumn{3}{|l|}{ Original department } \\
\hline Infectious disease & $11.00(6.50 \sim 17.50)$ & $6.00(6.00 \sim 14.00)$ \\
\hline Department of respiration & $7.00(5.00 \sim 7.00)$ & $8.00(5.75 \sim 9.00)$ \\
\hline ICU & $6.00(5.00 \sim 6.75)$ & $5.00(5.00 \sim 10.00)$ \\
\hline Other & $10.00(5.75 \sim 17.00)$ & $12.00(8.00 \sim 14.75)$ \\
\hline $\mathrm{H}$ value & 7.42 & 7.713 \\
\hline$P$ value & 0.06 & 0.056 \\
\hline \multicolumn{3}{|l|}{ Time of anti epidemic } \\
\hline$<1$ week & $7.00(6.00 \sim 12.00)$ & $7.00(6.50 \sim 13.00)$ \\
\hline $1 \sim 2$ weeks & $6.00(5.00 \sim 11.00)$ & $8.00(5.00 \sim 11.50)$ \\
\hline$>2$ weeks & $8.00(5.00 \sim 11.00)$ & I \\
\hline $\mathrm{H}$ value & 0.198 & -0.579 \\
\hline$P$ value & 0.906 & 0.563 \\
\hline
\end{tabular}


symptoms among doctors and nurses exposed to H7N9 patients, and found that intermediate staff showed higher arousal symptoms than senior staff [20]. Jinghong, et al. also reported that the psychological health of medical and nursing staff in a fever outpatient clinic during the SARS epidemic was associated with their professional title [25]. Because of their agelimit, senior medical staffs are less equipped [25]; therefore, primary and intermediate medical staff take more responsibility in an epidemic and thus experience more psychological stress. Compared with senior medical staff, primary and intermediate medical staff also experience more stress about aspects other than professional work. Wang, et al. explored factors associated with occupational stress for medical staff in a tertiary general hospital [26]. Of 25 occupational stressors, those most associated with stress were substantial high-risk job responsibility and heavy workload. The high-risk nature of medical care not only includes people's limited understanding of disease, limitations of diagnosis and treatment methods, and differences in medical staff knowledge and skills, but also involves the risk of responsibility that must be taken during medical practice, and the unavoidable risk of being hurt in special work [27]. In addition, especially in this COVID-19 epidemic, intermediate level medical staffs are mainly involved in first line clinical treatment. Work intensity and rotation, and managing patient pain and distress [28], further negatively impact physical and mental health.

The correlation analysis showed that the severity of negative emotional symptoms was related to several factors: Disease hazards, intensity of work, uncertainty of working hours, concerns about safety and security, and mismanagement. Although there is a substantial research effort to clarify the transmission, pathogenesis, and treatment of COVID-19, its complexity and persistence remain unclear [29]. In addition, the inadequate application of information technology to try to resolve the crisis [30] may place greater psychological pressure on medical workers in direct contact with patients. The COVID-19 outbreak occurred during the annual Lunar New Year holiday in China. This had two important effects: Greater mobility of a large population and a lack of factory workers [31]. Therefore, personnel management and distribution of medical supplies are important challenges in the early stage of the epidemic.

Some limitations of this survey should be noted. First, we were unable to assess psychological conditions before the COVID-19 outbreak, and did not compare mental state between medical staff caring for patients with COVID-19 and other medical staff; it is therefore difficult to draw causal inferences. Second, the "other factors" that were assessed on the questionnaire were not defined, to reduce responder fatigue and time burden. Therefore we could not assess the correlation between psychological state and specific other factors. Finally, the relatively small sample size may have redu- ced the statistical power and produced false negative results.

\section{Conflict of Interest}

None.

\section{Author Statement Contributors}

Guo Zhongwei conceptualized and designed the study, drafted the initial manuscript and revised, and approved the final manuscript as submitted. Wang Lijuan and Lu Longxi designed the data collection instruments, they made equal contributions. Zheng Jisheng, Zhu Ruichen coordinated and supervised data collection. Liu Xiaozheng carried out the initial analyses, and interpreted the data.

\section{Role of the Funding Source}

None.

\section{Participants}

All participants met the following criteria: Drawn from 105 medical units in Zhejiang Province, working on the clinical front line in Tianyou Hospital affiliated to Wuhan University of Science and Technology, self-isolating after work in the same apartment hotel located 500 meters from Tianyou Hospital. The study was approved by the ethics committee of Tongde Hospital in Zhejiang Province. All participants provided informed consent.

\section{Acknowledgment}

We thank Diane Williams, PhD, from Liwen Bianji, Edanz Editing China (www.liwenbianji.cn/ac), for editing the English text of a draft of this manuscript.

\section{References}

1. Wu Z, McGoogan JM (2020) Characteristics of and important lessons from the coronavirus disease 2019 (COVID-19) outbreak in China: Summary of a report of 72314 cases from the Chinese Center for Disease Control and Prevention. JAMA.

2. Ping Wu, Yunyun Fang, Zhiqiang Guan, Bin Fan, Junhui Kong, et al. (2009) The psychological impact of the SARS epidemic on hospital employees in China: Exposure, risk perception, and altruistic acceptance of risk. Can J Psychiatry $54: 302-311$.

3. Wang LW, Yang LH, Chen XB (2003) The investigation of psychological status of medical staff during epidemic outbreak stage of SARS in Wuhan. Chin J Behav Med Sci 12: 556-558.

4. Gan J, Li X, Zhang W (2004) Relative factors of mental health of anti-SARS medical staff. Qianwei Journal of Medicine 26

5. Renjie Zhang, Tingting Jiang, Na Li, Zhen Wang, Biyao Liu, et al. (2015) The negative psychology for the public in Zhejiang province during the epidemic of human H7N9 avian influenza. Chinese Journal of Preventive Medicine 49: 1073-1079.

6. Xin Tong, Dongmei An, Aileen McGonigal, Sung-Pa Park, Dong Zhou (2016) Validation of the generalized anxiety disorder-7 (GAD-7) among Chinese people with epilepsy. Epilepsy Res 120: 31-36. 
7. Wenzheng Wang, Qian Bian, Yan Zhao, Xu Li, Wenwen Wang, et al. (2014) Reliability and validity of the Chinese version of the patient health questionnaire (PHQ-9) in the general population. Gen Hosp Psychiatry 36: 539-544.

8. Li Li, Changli Wan, Ru Ding, Yi Liu, Jue Chen, et al. (2015) Mental distress among liberian medical staff working at the China ebola treatment unit: A cross sectional study. Health Qual Life Outcomes 13: 156.

9. Dong Ji, Ying Jie Ji, Xue Zhang Duan, Wen Gang Li, Zhi Qiang Sun, et al. (2017) Prevalence of psychological symptoms among ebola survivors and healthcare workers during the 2014-2015 ebola outbreak in Sierra Leone: A cross-sectional study. Oncotarget 8: 12784-12791.

10. Liu L, Zhang X, Zhang X (2014) Correlation of mental health level and trait coping styles in the frontline medical staff fighting for Ebola. Chin J Mod Nurs 20: 4411-4412.

11. Ying Li, Huan Wang, Xu Rui Jin, Xiang Li, Michelle Pender, et al. (2018) Experiences and challenges in the health protection of medical teams in the Chinese ebola treatment center, Liberia: A qualitative study. Infect Dis Poverty 7: 92.

12. Chunzi Liu, Huaming Wang, Lin Zhou, Hui Xie, Huiyin Yang, et al. (2019) Sources and symptoms of stress among nurses in the first Chinese anti-Ebola medical team during the Sierra Leone aid mission: A qualitative study. Int J Nurs Sci 6: 187-191.

13. Tam CWC, Pang EPF, Lam LCW, Chiu HFK (2004) Severe acute respiratory syndrome (SARS) in Hong Kong in 2003: Stress and psychological impact among frontline healthcare workers. Psychol Med 34: 1197-1204.

14. Panagiota Goulia, Christos Mantas, Danai Dimitroula, Dimitrios Mantis, Thomas Hyphantis (2010) General hospital staff worries, perceived sufficiency of information and associated psychological distress during the $\mathrm{A} / \mathrm{H} 1 \mathrm{~N} 1$ influenza pandemic. BMC Infect Dis 10: 322.

15. Li X, Yang Y, Hou W (2010) Investigation of the mental health of the medical staffs during the influenza $A(\mathrm{H} 1 \mathrm{~N} 1)$ prevalent period. BMJ 7.

16. Shuai Liu, Lulu Yang, Chenxi Zhang, Yu Tao Xiang, Zhongchun Liu, et al. (2020) Online mental health services in China during the COVID-19 outbreak. Psychiatry 7: 17-18.

17. Robin Goodwin, Shamsul Haque, Felix Neto, Lynn B Myers (2009) Initial psychological responses to Influenza A, H1N1 ("Swine flu"). BMC Infect Dis 9: 166.

18. Zhou Xu, Shu Li, Shen Tian, Hao Li, Ling-quan Kong (2020) Full spectrum of COVID-19 severity still being depicted. The Lancet 395: 947-948.
19. Shwu Hua Lee, Yeong Yuh Juang, Yi Jen Su, Hsiu Lan Lee, Yi Hui Lin, et al. (2005) Facing SARS: Psychological impacts on SARS team nurses and psychiatric services in a Taiwan general hospital. Gen Hosp Psychiatry 27: 352358.

20. Liling Tang, Lingling Pan, Liping Yuan, Lei Zha (2017) Prevalence and related factors of post-traumatic stress disorder among medical staff members exposed to H7N9 patients. Int J Nurs Sci 4: 63-67.

21. Kunitaka Matsuishi, Ayako Kawazoe, Hissei Imai, Atsushi Ito, Kentaro Mouri, et al. (2012) Psychological impact of the pandemic (H1N1) 2009 on general hospital workers in Kobe. Psychiatry Clin Neurosci 66: 353-360.

22. Lau JTF, Griffiths S, Choi KC, Tsui HY (2010) Avoidance behaviors and negative psychological responses in the general population in the initial stage of the $\mathrm{H} 1 \mathrm{~N} 1$ pandemic in Hong Kong. BMC Infectious Diseases 10: 139.

23. Holbrook TL, Hoyt DB, Stein MB, Sieber WJ (2002) Gender differences in long-term posttraumatic stress disorder outcomes after major trauma: Women are at higher risk of adverse outcomes than men. J Trauma 53: 882-888.

24. Lau JTF, Tsui H, Kim JH, Griffiths S (2006) Perceptions about status and modes of $\mathrm{H} 5 \mathrm{~N} 1$ transmission and associations with immediate behavioral responses in the Hong Kong general population. Prev Med 43: 406-410.

25. Jinghong L, Wei W, Wenbin G (2004) A survey of psychological health state of medical and nursing staffs of fever out-patient clinic in Military Hospitals during SARS epidemic period and analysis of its related factors.

26. Wang X, Hua L, Bai H (2010) An analysis of characteristic occupational stressors for medical staff in general hospitals. CJHA 26: 257-262.

27. Feng $T$, Zhao L, Zou $L$ (1996) Reflection on the issue of doctors' practice circumstances. CJHA.

28. Kang L, Li Y, Hu S, Chen M, Yang C, et al. (2020) The mental health of medical workers in Wuhan, China dealing with the 2019 novel coronavirus. Psychiatry 7.

29. Xu Z, Shi L, Wang Y, Zhang J, Huang L, et al. (2020) Pathological findings of COVID-19 associated with acute respiratory distress syndrome. Lancet Respir Med 8: 420-422.

30. Wang C, Cheng Z, Yue XG, McAleer M (2020) Risk management of COVID-19 by Universities in China. J Risk Financial Manag 13: 36.

31. Chen S, Yang J, Yang W, Wang C, Barnighausen T (2020) COVID-19 control in China during mass population movements at new year. Lancet 395: 764-766. 